\title{
The Quantitative Prediction of Pipeline Cracks Using Metal Magnetic Memory Based on a Regression Model
}

\author{
Lihong Gong ${ }^{*}, 1,2$, Zhuxin $\mathrm{Li}^{1}$ and Zhiqiang Song ${ }^{1}$ \\ ${ }^{I}$ Department of Military Oil Supply Engineering, Logistic Engineering University of PLA, Chongqing 401331, China \\ ${ }^{2}$ Department of Military Electric Power Engineering, Chongqing Communication Institute, Chongqing 400035, China
}

\begin{abstract}
The technique of metal magnetic memory (MMM) has great advantages in detecting early failures such as stress concentration and fatigue damage of ferromagnetic components, which has been widely applied due to its high efficiency, low requirements for surface preparation and ease of operation. However, research into the quantitative description of defect characteristics is still far from adequate. To promote relative study in this area, in this paper, a regression model is employed to analyze the sizes of surface cracks in pipelines. Three nonlinear functions are obtained to predict the length, width and depth of cracks respectively based on a regression model. Length prediction is convenient and accurate, with the average coefficient of determination of training samples up to 0.994 and that of testing samples 0.962 . Moreover, as the width and depth are small (less than $2 \mathrm{~mm}$ ), the accuracy of size prediction is very high. The obtained functions provide a useful method of predicting the crack sizes of pipelines according to MMM signals.
\end{abstract}

Keywords: Metal magnetic memory, pipeline crack, regression model.

\section{INTRODUCTION}

Some methods such as low-plasticity ball burnishing can improve the fatigue strength of steel components [1], but faults are frequent in metal pipelines because of long term usage or high pressure, so it is necessary to examine the pipelines regularly by nondestructive testing (NDT) methods. Nondestructive testing means the use of noninvasive techniques to determine the integrity of a material, component or structure or quantitatively measure some characteristic of an object. The most often used NDT methods include ultrasonic testing, radiographic testing, penetrant testing, eddy current testing and magnetic particle testing [2]. Among various NDT techniques, lots of nondestructive magnetic techniques have been extensively adopted to ensure the operating safety of ferromagnetic structures and components in engineering such as magnetic particle testing (MPT), eddy current testing (ECT), magnetic flux leakage (MFL), magnetic Barkhausen noise (MBN), magneto acoustic emission (MAE) and recently developed metal magnetic memory (MMM). These techniques utilize the inherent ferromagnetic properties of the steels for nondestructive evaluation of a wide range of material mechanical properties [3]. As we all know, MFL, MBN and MMM are three kinds of popular magnetic NDT technologies. Among them, MFL and MBN techniques may be ascribed to active magnetic test methods which require applying a strong artificial field to magnetize the tested objects. Thus, the two techniques are usually time-consuming and even impractical for some irregular structures. For the purpose of developing

*Address correspondence to this author at the Department of Military Oil Supply Engineering, Logistic Engineering University of PLA, Chongqing 401331, China; Tel: +86-13983678633; E-mail: glh1130@aliyun.com a more simple and effective NDT magnetic technique to meet the requirements in engineering, the passive magnetic technique has been paid attention to in the recent years.

As a new kind of passive magnetic flux leakage nondestructive testing technique, MMM testing technology was first put forward by a Russian expert in 1997 which is based on the mechanism of the cooperative action of the magnetoelasticity and magneto-mechanical effect. MMM testing can be used to detect not only the stress concentration area for the ferromagnetic metal material but also all kinds of microscopic and macroscopic cracks which are caused by stress concentration. Unlike traditional magnetic testing methods such as MFL, the geomagnetic field instead of an external magnetic field is applied in MMM as the stimulus source. In MMM techniques, what is measured are the self-magnetic flux leakage signals of ferromagnetic. Under the joint effect of the geomagnetic field and the applied load, ferromagnetic signals are generated in the stress concentration zones. According to experimental studies, the tangential component of MMM testing, $H_{p}(x)$, reaches to maximum value, as well as the normal component of MMM testing, $H_{p}(y)$, passes through zero and changes its polarity in the stress concentration zones. This magnetic state is retained even if the applied load is removed. Therefore, MMM techniques are normally used to detect the stress concentration zones, to predict the residual lifetime of ferromagnetic components and to diagnose the early damages, etc.. MMM technique displays a lot of attractive advantages as follows: (1) MMM testing technology is effective in characterizing the early damages of ferromagnets, especially the micro-damage due to local stress concentration. (2) It is easyoperation, high-efficiency, simple criteria and has faster measuring velocity than other NDT methods. (3) The MMM technique is suitable for many engineering inspection, 
evaluation and diagnosis of faults in the gas or oil pipelines, turbines, wheels, pressure vessels, rails and so on [4-10]. But as a novel magnetic testing method, there are still many important problems about MMM testing which need to be studied, such as the further theoretical study of the magnetic memory phenomenon mechanism, the best defect size range of MMM testing, the quantitative relationship between defect sizes and MMM signals. At present, MMM testing is unable to directly determine defect sizes [11-13]. That is to say, the sizes of defects need to be predicted accurately. Some researchers have reported the qualitative relationship between defect sizes and MMM testing signals $[14,15]$. K. Yao et al. defined and analyzed some parameters on the defect width, depth, location, testing position and direction. They promoted the development of MMM from a qualitative technique to a quantitative NDT technique [16]. Dong et al. found an approximate linear relationship according to their MMM signals and crack length [17].

Generally, cracks are common defects in oil and gas pipelines. This paper presents a mathematical model to predict the sizes of cracks according to their signals of MMM. As is known, different cracks have different MMM signals. The crack profile and its parameters can be determined by the information contained in the MMM signals [18]. Therefore, the sizes of cracks can be predicted according to MMM signals by appropriate methods. Actually, rectangular cracks are common in the cracks of pipelines. Hence, rectangular cracks are taken as the research object. In short, this paper aims to present a useful quantitative model about rectangular crack sizes such as length, depth and width based on MMM signals by linear and nonlinear regression. Consequently, the forecasting fitting functions of the sizes of pipeline cracks are obtained.

\section{EXPERIMENTS AND DATASETS}

\subsection{Experimental details}

UGY-94 oil pipelines were used as the basic detected objects in the experiments, while different cracks with different sizes were made artificially. UGY-94 pipeline is made of material for X60JY strip steel, whose chemical composition and mechanical properties are shown in Tables 1 and 2.

First, the pipeline sections were split into experiment test pieces, using a pipe cutter, and a series of different cracks with different size were made, as much as possible close to the actual situation in a pipeline. To control the precision and machining precisions of the cracks, the electric spark machining technology was used. These artificial cracks were simulated as the real cracks in the pipelines. Fig. (1) presents one of the schematic diagrams and photos of test pieces with pre-made cracks. Then, aging treatment was then carried out on the test pieces to eliminate residual stress generated in machining process. Finally, the signals of the test pieces were detected by MMM testing, one by one, on the experimental platform. Three scanning lines were selected with a length of $290 \mathrm{~mm}$, indicated by a, b and c.

The static tension tests were carried out on a RGM-4100 electronic tensile testing machine, whose static load error is $0.5 \%$ to $1 \%$ and the maximum test force is $100 \mathrm{kN}$. The experiment test piece was loaded at approximately $0.5 \mathrm{kN} / \mathrm{s}$ until reached to the designed load value. Then the test piece was taken from the tensile machine and laid on the detection platform. The MMM signals were detected along scanning lines by the MFL-4032A, a magnetic flux leakage/magnetic memory detector, which was a joint venture by Logistic Engineering University and Xiamen Eddysun Electronic Company. MFL-4032A is suitable for $150 \mathrm{~mm}$ diameter pipeline, which includes the magnetic flux leakage detection module, the magnetic memory detection module, the signal processing module, driver module and battery power supply module. After detection, the test piece reloaded to a higher value, and the above procedure was repeated.

(a) Schematic diagram of test piece with pre-made cracks

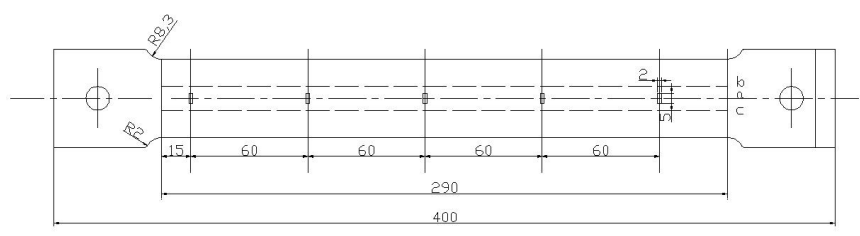

(b) Photo of test piece with pre-made cracks

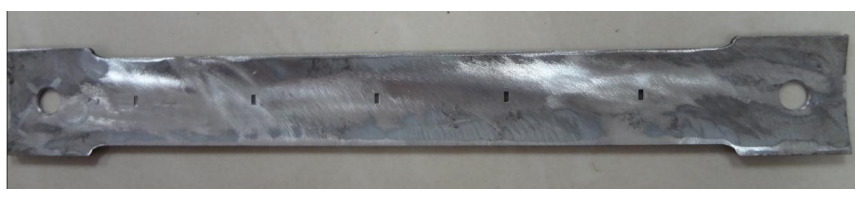

Fig. (1). Schematic diagram and photo of test piece with pre-made cracks.

\subsection{Dataset}

50 cracks in test pieces were designed in total. There are five kinds of crack length containing 5 samples of $5 \mathrm{~mm}, 10$

Table 1. Chemical Composition of X60 Pipeline Steel

\begin{tabular}{|c|c|c|c|c|c|c|c|c|}
\hline Steel & C & S & Mn & V & Si & P & Nb & Ti \\
\hline \hline X60 pipeline steel & $0.040-0.060$ & $\leq 0.010$ & $1.240-1.300$ & $0.015-0.030$ & $\leq 0.400$ & $\leq 0.020$ & $0.040-0.050$ & $0.006-0.008$ \\
\hline
\end{tabular}

Table 2. Mechanical Properties of X60 Pipeline Steel

\begin{tabular}{|c|c|c|c|c|c|}
\hline Steel & Elastic Modulus (GPa) & Tensile Strength (Mpa) & Compressive Strength (MPa) & Yield Strength (MPa) & Poisson Ratio \\
\hline \hline X60 pipeline steel & 200 & 520 & 496 & 420 \\
\hline
\end{tabular}


$\mathrm{mm}, 15 \mathrm{~mm}$ respectively, 6 samples of $20 \mathrm{~mm}$ and 29 samples of $25 \mathrm{~mm}$. Five kinds of crack width were designed including 5 samples of $0.5 \mathrm{~mm}, 1.5 \mathrm{~mm}$ and $2 \mathrm{~mm}$ respectively, 31 samples of $1 \mathrm{~mm}$ and 4 samples of $2.5 \mathrm{~mm}$. Crack depth included 10 samples of $0.5 \mathrm{~mm}, 1 \mathrm{~mm}, 1.5 \mathrm{~mm}$, $2 \mathrm{~mm}$ and $2.5 \mathrm{~mm}$ respectively.

By MMM testing, original MMM signals were obtained for every crack. Then, to facilitate analysis and modeling, the signal was used to derive 11 new indices to predict the sizes of the cracks, as explained below.

$x_{1}$ : The peak value of MMM original signals in $\mathrm{x}$ axis;

$x_{2}$ : The peak-to-peak value of MMM original signal in $\mathrm{x}$ axis;

$x_{3}$ : The peak value of MMM original signals in y axis;

$x_{4}$ : The peak-to-peak value of MMM original signal in $\mathrm{y}$ axis;

$x_{5}$ : The peak value of differential gradient of detection original signal in $\mathrm{x}$ axis;

$x_{6}$ : The peak-to-peak value of differential gradient of detection original signal in $\mathrm{x}$ axis;

$x_{7}$ : The peak-to-peak value of differential gradient of detection original signal in y axis;

$x_{8}$ : The energy of MMM original signal in $\mathrm{x}$ axis;

$x_{9}$ : The energy of MMM original signal in y axis;

$x_{10}$ : The differential gradient energy of detection original signal in $\mathrm{x}$ axis;

$x_{11}$ : The differential gradient energy of detection original signal in y axis.

\section{MODELING AND ANALYSIS}

\subsection{The Predicting Model of the Length}

$x_{2}, x_{3}, x_{4}, x_{5}, x_{6}, x_{7}, x_{10}$ are selected by stepwise regression as the variables to predict the length of cracks. All samples are randomly divided into training set and testing set for 100 times to simulate with regression, where there are 4 samples with the length of cracks of $5 \mathrm{~mm}, 10 \mathrm{~mm}, 15 \mathrm{~mm}, 20 \mathrm{~mm}$ separately and 20 samples with the length of $25 \mathrm{~mm}$. The rest of the 14 samples are classified as testing set.

By running linear regression simulation one hundred times, 100 coefficients of determination can be obtained for training samples, in which the maximum is 0.982 , the minimum is 0.958 , and the average is 0.968 . As to testing samples, the maximum coefficient of determination is 0.984 , the minimum is 0.680 and the average is 0.938 (Fig. 2a).

In order to improve the accuracy of length prediction, some nonlinear terms are included to regress. First, to establish the relation between the length of cracks and the indices, indices with crack length of $5 \mathrm{~mm}, 10 \mathrm{~mm}, 15 \mathrm{~mm}$, $20 \mathrm{~mm}$ and $25 \mathrm{~mm}$ respectively are averaged. Then, by curve fitting, it is found that the length corresponds to the square of $x_{7}$ and the cube of the other variables (Fig. 3). Therefore, the length of cracks could be determined by some of the (a) Linear model

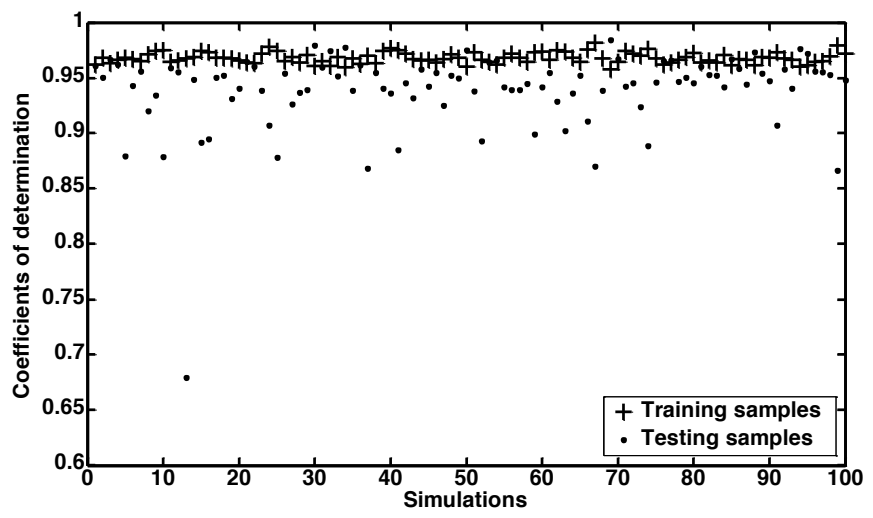

(b) Nonlinear model

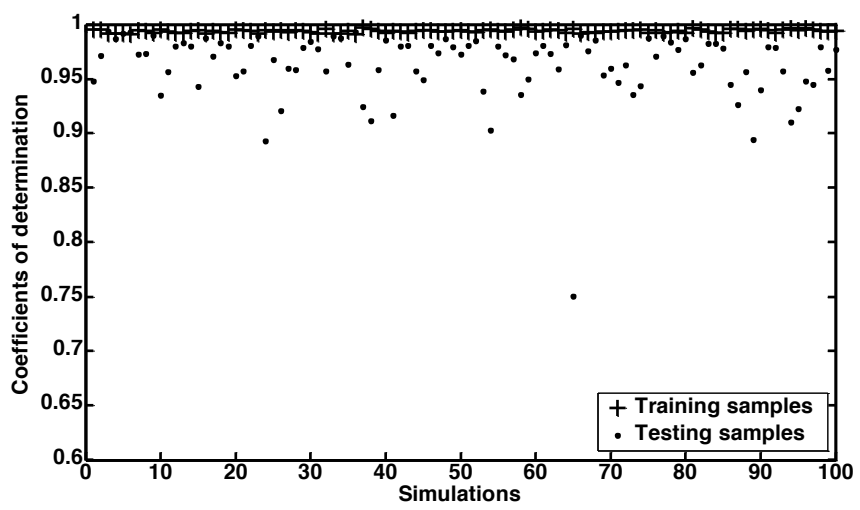

Fig. (2). The coefficients of determination in the 100 regression simulations to the length of cracks.

variables $x_{2}, x_{3}, x_{4}, x_{5}, x_{6}, x_{7}, x_{10}, x_{2}{ }^{2}, x_{3}{ }^{2}, x_{4}{ }^{2}, x_{5}{ }^{2}, x_{6}{ }^{2}, x_{7}{ }^{2}, x_{10}{ }^{2}$, $x_{2}{ }^{3}, x_{3}{ }^{3}, x_{4}{ }^{3}, x_{5}{ }^{3}, x_{6}{ }^{3}$ and $x_{10}{ }^{3}$. So, finally, stepwise regression is used to screen them and the variables $x_{2}, x_{3}, x_{4}, x_{5}, x_{6}, x_{7}$, $x_{2}{ }^{2}, x_{3}{ }^{2}, x_{5}{ }^{3}$ are screened out. These indices are used to make the regression. Accordingly, the maximum coefficient of determination of training samples is 0.997 , the minimum is 0.991 and the average is 0.994 . With regard to the testing samples, the maximum coefficient of determination is 0.993 , the minimum is 0.750 , and the average is 0.962 (Fig. 2b).

Fig. (2) shows that the result of nonlinear model is better than that of linear model. Furthermore, the difference of coefficients of determination of the training samples between linear model and nonlinear model is tested by independent sample $\mathrm{t}$ test, $t=-51.631, p=0$. The same result of testing samples is $t=-4.916, p=1.853 \times 10^{-6}$. For Both training samples and testing samples, the nonlinear model is significantly better than the linear model. Therefore, one of the good results, which is neither the best nor the worst, in the 100 simulations is chosen as the length prediction model of cracks. The fitting function is

$$
\begin{aligned}
& L=-0.002 x_{5}^{3}+19.527 x_{3}^{2}-5.643 x_{2}^{2}+234.300 x_{7}+487.990 x_{6} \\
& -315.030 x_{5}+2685.600 x_{4}-2828.800 x_{3}-254.340 x_{2}+22.585
\end{aligned}
$$

where $F=504.020, p=0$. The coefficient of determination of training samples is 0.995 and that of testing samples is 0.971 . 

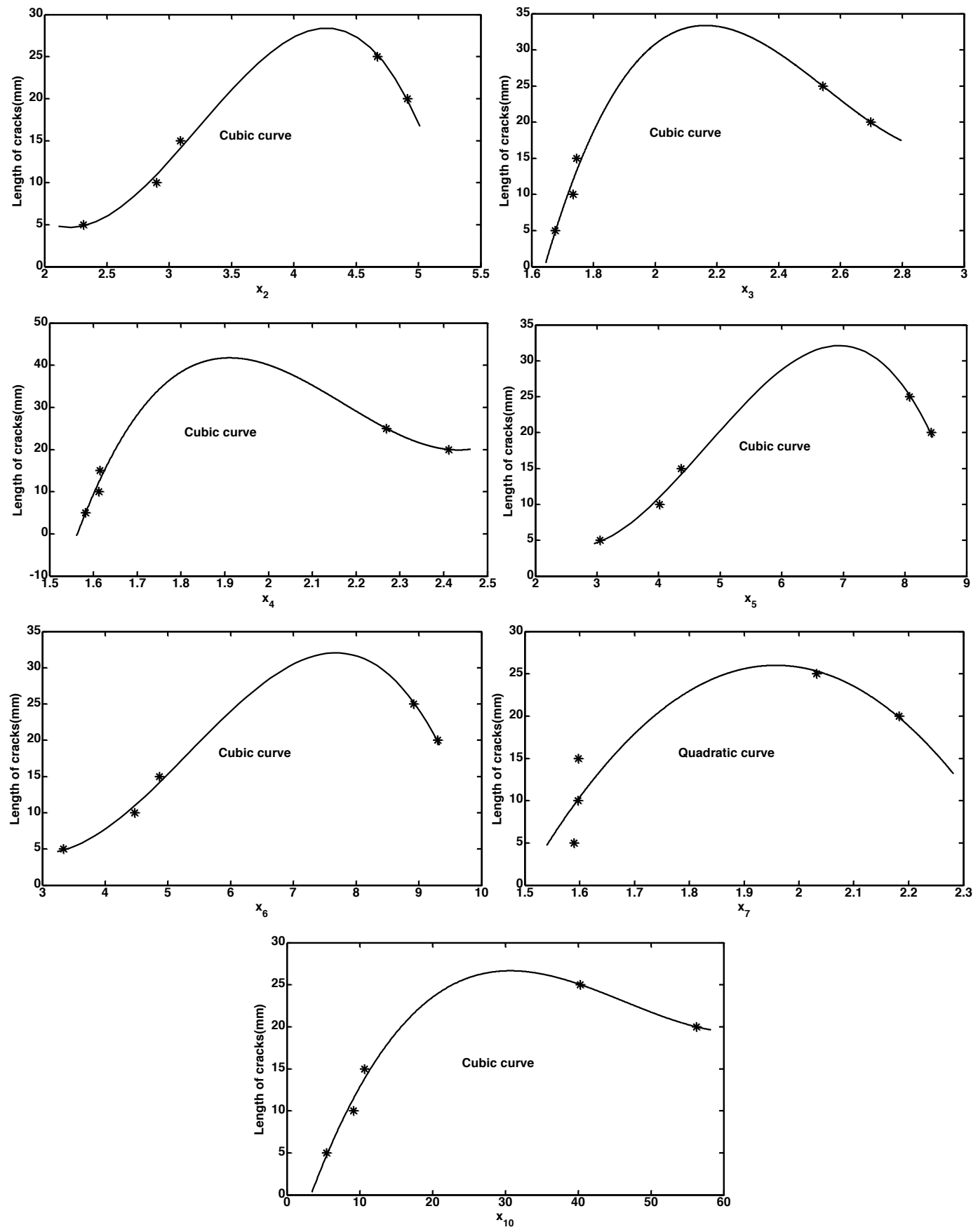

Fig. (3). The fitting curves between the length of cracks and the indices.

\subsection{The Predicting Model of the Width}

Stepwise regression selected $x_{4}, x_{6}$ and $x_{7}$ as the variables to predict the width of cracks. 4 samples with crack width of $0.5 \mathrm{~mm}, 1.5 \mathrm{~mm}$ and $2 \mathrm{~mm}$ separately, 26 samples with crack width of $1 \mathrm{~mm}$ and 3 samples with crack width of 2.5 $\mathrm{mm}$ are randomly divided into training sample set and the rest of 9 samples are divided into testing sample set. After 100 linear regression simulations, 100 coefficients of determination can be gained, in which the maximum is 0.674 , the minimum is 0.286 and the average is 0.459 in training samples, and the maximum, minimum and average in testing samples are $0.686,0.148$ and 0.422 respectively (Fig. 4a).
Fig. (4a) shows that the result of linear model is unsatisfactory. Therefore, in an attempt to improve prediction accuracy, nonlinear terms are joined. The results of curve fitting reflect the relation between the width of the cracks and the indices $x_{4}, x_{6}$ and $x_{7}$ (Fig. 5). Obviously, all or some of the biquadratic, cubic, quadratic and linear terms of these indices could be the determinant of the width of cracks, which should be screened by stepwise regression. The terms $x_{4}{ }^{4}, x_{6}{ }^{4}, x_{7}{ }^{4}, x_{6}{ }^{3}$, $x_{7}{ }^{3}, x_{7}^{2}, x_{4}, x_{6}$ and $x_{7}$ are selected. Finally, they are used to make a regression simulation. In the 100 times of simulations, the maximum, minimum and average coefficients of determination of training samples are $0.919,0.723$ and 0.778 , and those of testing samples are $0.831,0.113$ and 0.492 separately (Fig. 4 b). 
(a) Linear model

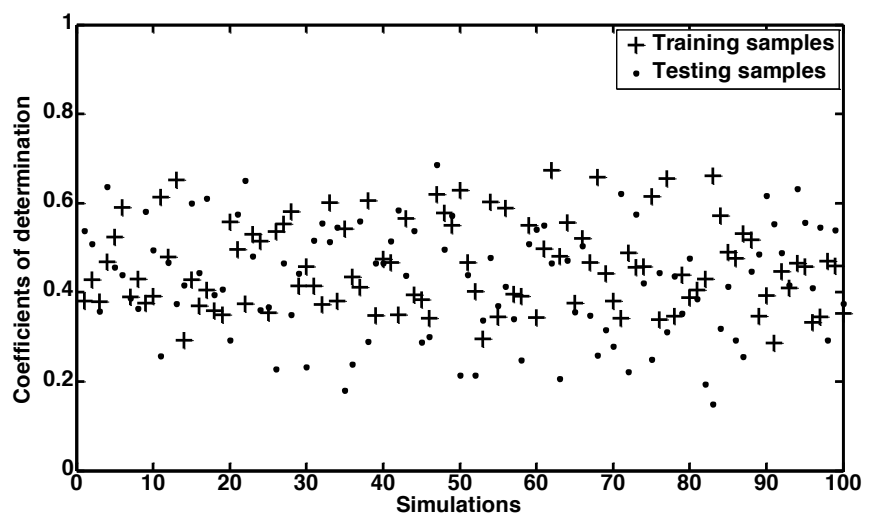

(b) Nonlinear model

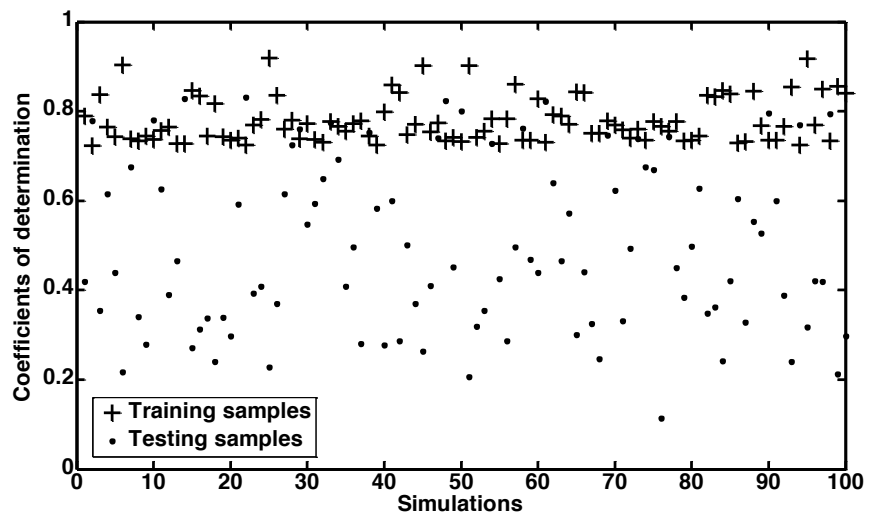

Fig. (4). The coefficients of determination in the 100 regression simulations to the width of cracks.

The difference of coefficients of determination between linear model and nonlinear model is tested. Concerning training samples, $t=-29.091, p=0$. Regarding the testing samples, $t=-3.080, p=0.002$. The coefficient of determination of nonlinear model is higher than that of linear model, so that one result is chosen to predict the width of cracks. Its fitting function is

$$
\begin{aligned}
& W=-0.029 x_{4}^{4}+0.0002 x_{6}^{4}-0.388 x_{7}^{4}-0.007 x_{6}^{3}+7.367 x_{7}^{3} \\
& -33.115 x_{7}^{2}-34.961 x_{4}-0.261 x_{6}+62.136 x_{7}-11.956
\end{aligned}
$$

where $F=8.407, \quad p=1.960 \times 10^{-6}$. The coefficients of determination of training samples and testing samples are 0.783 and 0.727 .

\subsection{The Predicting Model of the Depth}

Stepwise regression is used to screen the important indices of depth of cracks and the indices are $x_{7}, x_{8}, x_{10}$ and $x_{11}$. There are five kinds of depth of cracks. 8 samples of each kind of depth of cracks are randomly divided into training set, while the other 10 samples are divided into testing set. In the 100 linear regression simulations, the maximum, minimum and average coefficients of determination of training samples are 0.826 , 0.728 and 0.771 , and those of testing samples are $0.928,0.364$ and 0.715 (Fig. 6a).

In order to improve the prediction accuracy, nonlinear terms can be added. By curve fitting, the relation between the depth of the cracks and the indices can be obtained (Fig. 7). So, some of the indices $x_{7}, x_{8}, x_{10}, x_{11}, x_{8}^{2}, x_{10}^{2}, x_{11}^{2}, x_{7} x_{8}$, $x_{7} x_{10}, x_{7} x_{11}, x_{8} x_{10}, x_{8} x_{11}, x_{10} x_{11}$ and $x_{10}{ }^{3}$ could determine the depth of cracks. Stepwise regression is used to screen them and $x_{7}, x_{8}, x_{10}$ and $x_{7} x_{11}$ are screened out. Eventually, the four selected indices are used to make regression simulation.

For the 100 simulations, the maximum, minimum and average coefficients of determination of training samples are $0.970,0.878$ and 0.903 , and those of testing samples are 0.968 , 0.339 and 0.781 respectively. The results are shown in Fig. (6b).
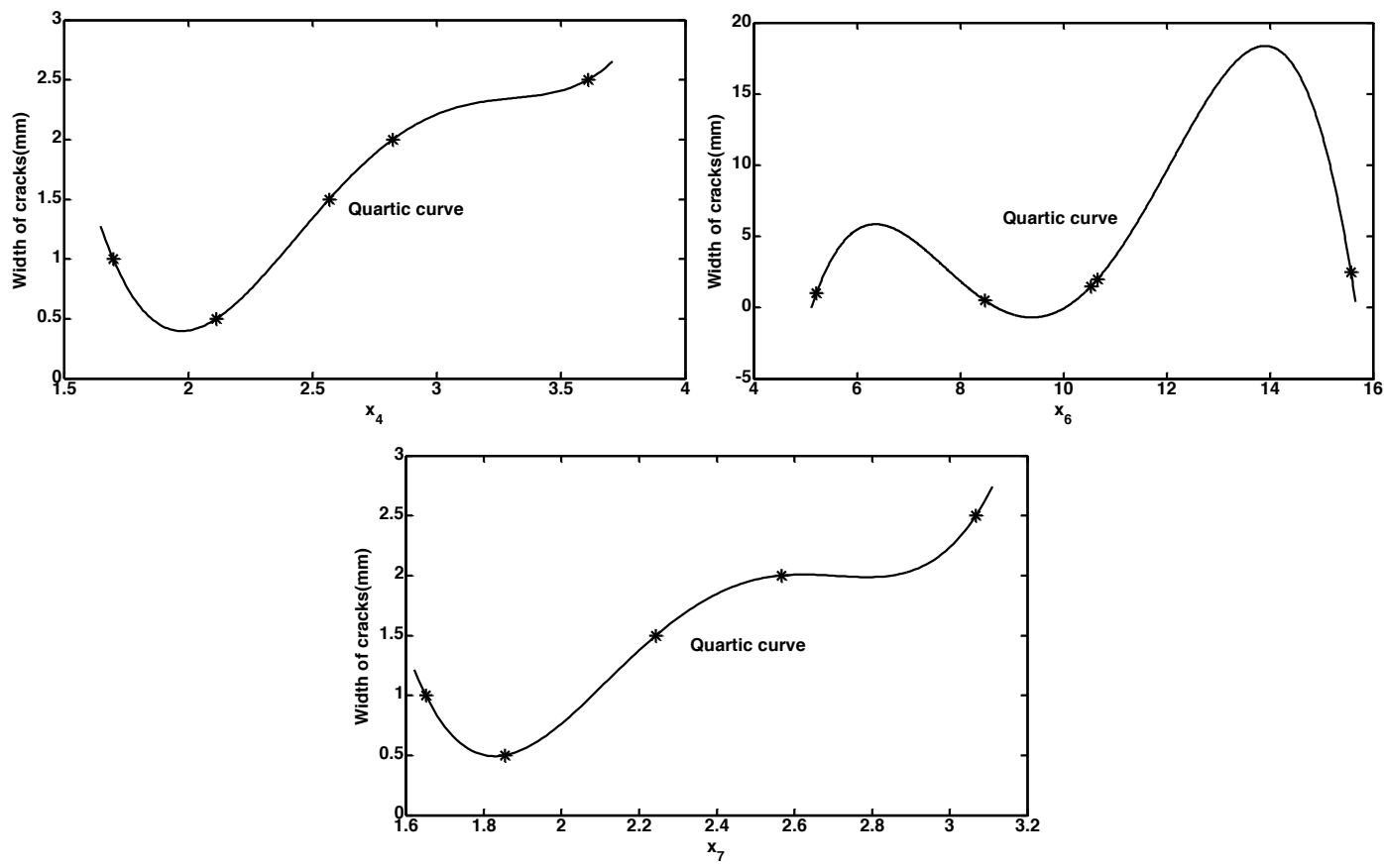

Fig. (5). The fitting curves between the width of cracks and the indices. 
(a) Linear model

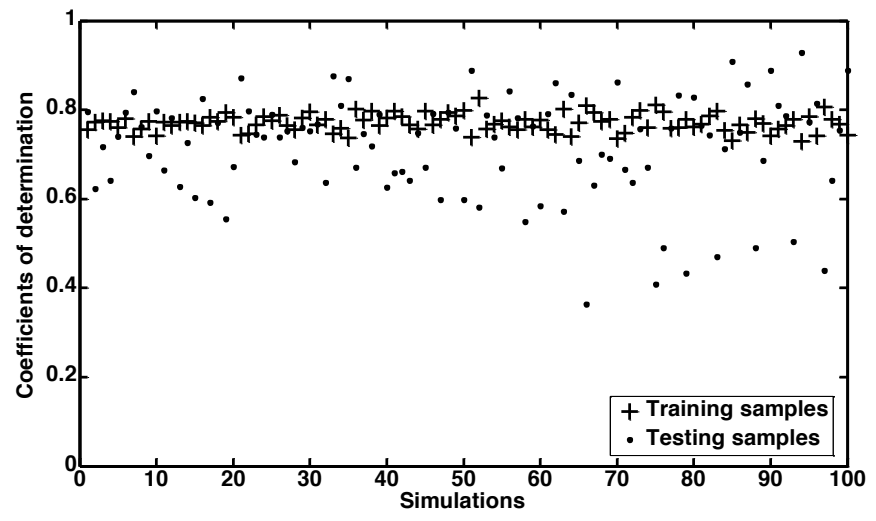

(b) Nonlinear model

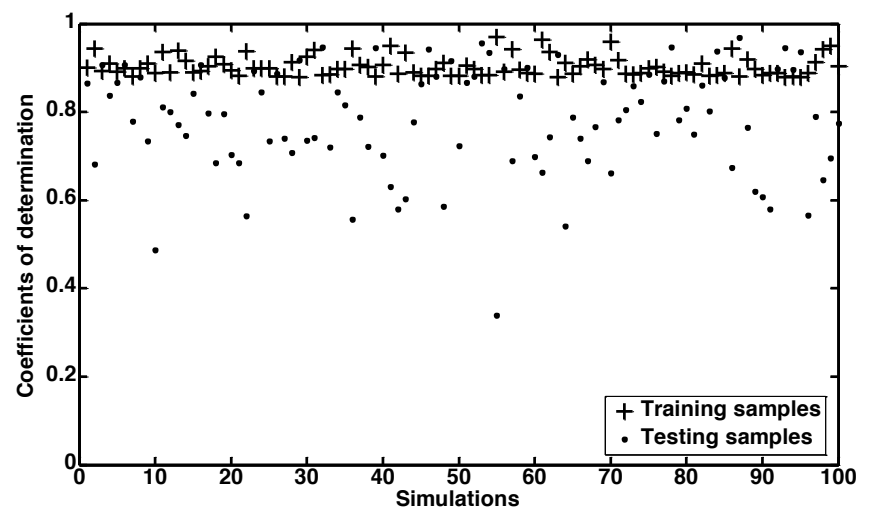

Fig. (6). The coefficients of determination in the 100 regression simulations to the depth of cracks.

In the end, the difference of coefficients of determination between linear model and nonlinear model is tested: $t=-44.309, p=0$ in training samples and $t=-3.870, p=0.0002$ in testing samples. The coefficient of determination of
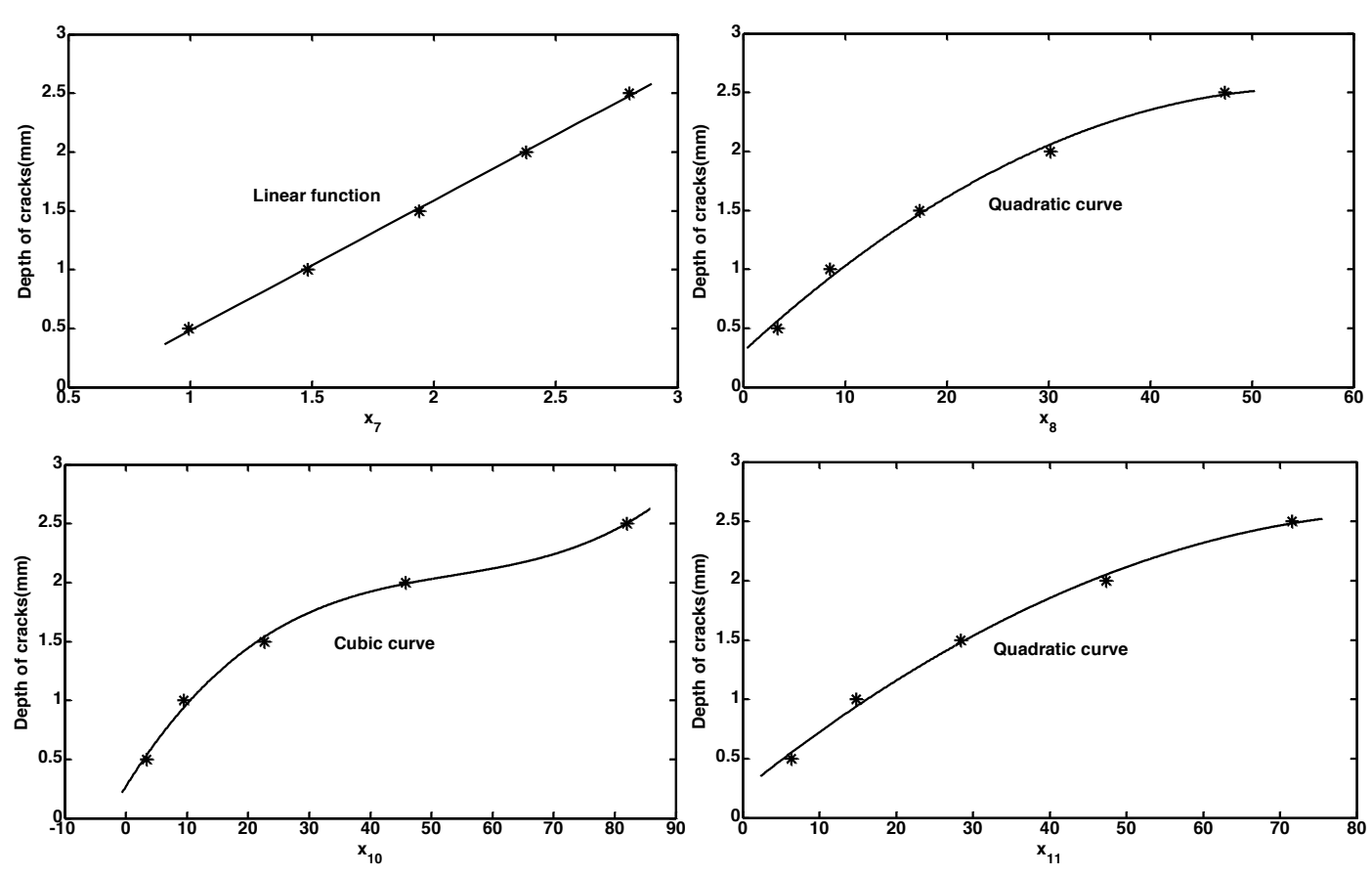

Fig. (7). The fitting curves between the depth of cracks and the indices.

nonlinear model is significantly higher than that of linear model. Therefore, we can choose one of the good results as the depth predicting model, whose fitting function is

$$
\mathrm{D}=0.007 x_{7} x_{11}+0.072 x_{7}-0.255 x_{8}+2.996 x_{10}-1.821
$$

where $F=16.210, p=3.627 \times 10^{-9}$. The coefficients of determination of training samples and testing samples are 0.901 and 0.966 .

\subsection{Error analysis}

Functions (1), (2) and (3) can be used to predict the sizes of cracks. To all of the 50 samples, the coefficients of determination of length, width and depth are $0.989,0.753$ and 0.857 respectively. Their residuals are shown in Fig. (8). The average relative errors of length, width and depth are $3.582 \%, 16.012 \%$ and $17.897 \%$ respectively. The relative errors of length are very small. The average relative errors of width and depth are greater than those of length: the errors are great when the width is $2.5 \mathrm{~mm}$ and the depth is more than $2 \mathrm{~mm}$.

\section{CONCLUSIONS}

a. In this paper, firstly, we simply reviewed the three kinds of representative magnetic NDT techniques MFL, MBN and MMM, and summarized the advantages or research status of MMM. Then, using the MMM signals to predict the length, width and depth of cracks in oil pipelines. The results reveal that in the quantitative prediction of pipeline cracks, a nonlinear model is significantly better than a linear model.

b. The accuracy of length prediction is very high. As the width of crack is less than $2.5 \mathrm{~mm}$, the width can be predicted accurately. When the depth of a crack is greater than $2 \mathrm{~mm}$, the predicted results are relatively 
unreliable. The reason is that when the depth of a crack is too great (larger than $2 \mathrm{~mm}$ ), the pipe surface will shield the magnetic signal of cracks, so that the intensities of MMM signals decrease and predicted errors increase. Therefore, there is an effective range of cracks detected by MMM.

(a) Length

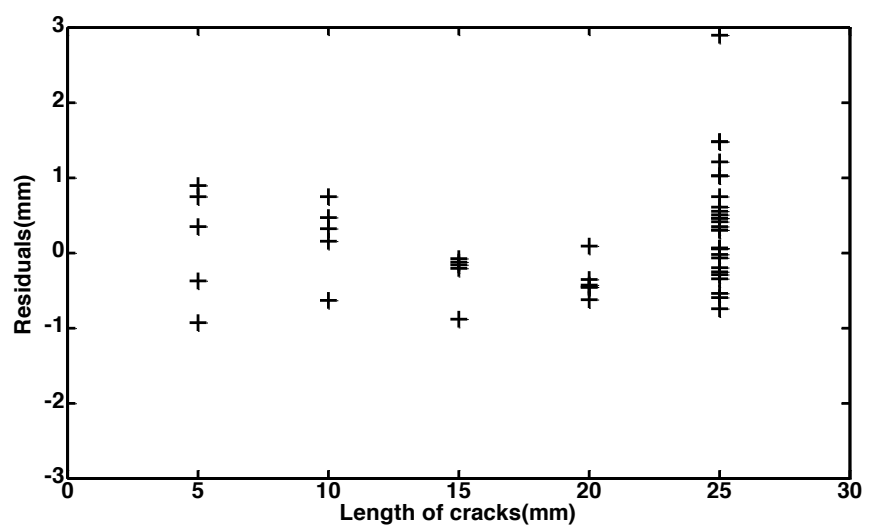

(b) Width

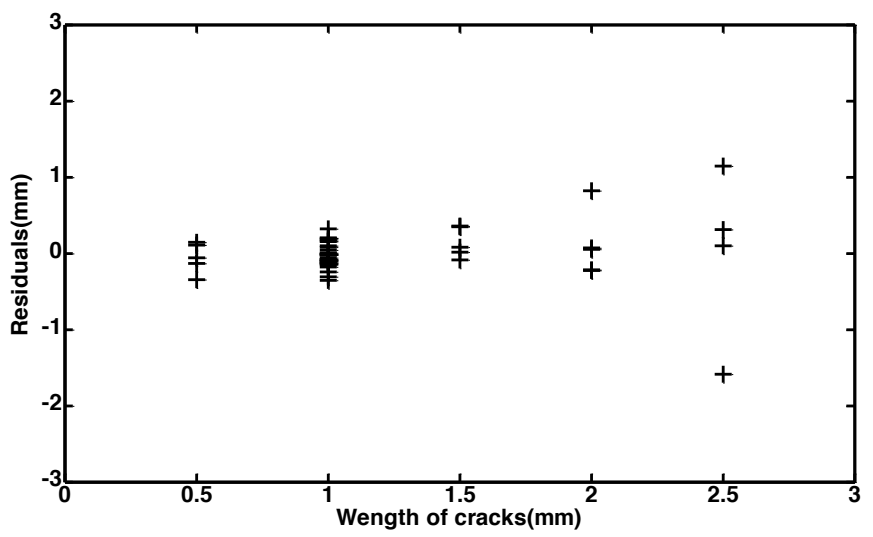

(c) Depth

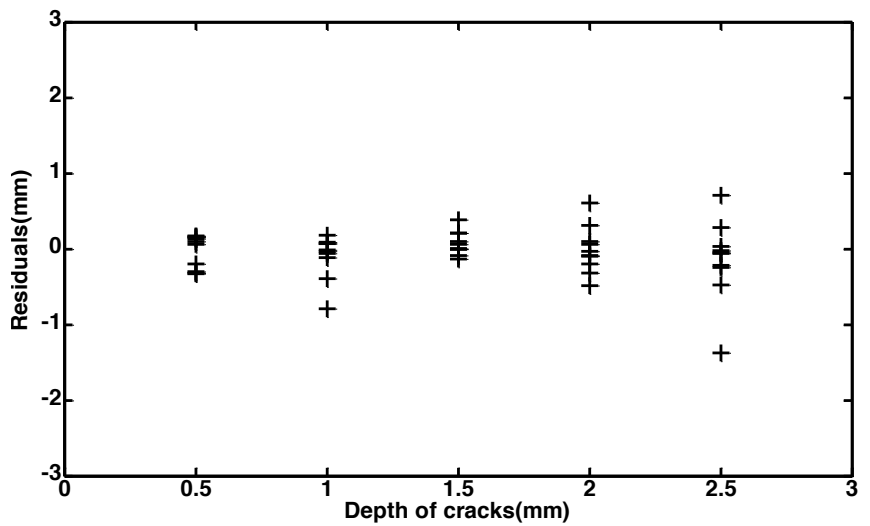

Fig. (8). The residuals of crack sizes prediction.

c. Here presented work provides useful information for predicting the sizes of cracks by MMM signals. However, some factors, such as the external magnetic fields, machining processes of ferromagnetic components, types of applied load, heat treatment of ferromagnetic components, will seriously affect the MMM signals. Therefore, means of excluding disturbing factors is of great importance to the accuracy of the quantitative study.

d. If more MMM testing data about pipelines' cracks become available in future studies, some new indices could be obtained according to original signals, which are closely related to the sizes of cracks, more accurate fitting regression functions would be obtained and this study would get more widely used in practical projects.

\section{CONFLICT OF INTEREST}

The authors confirm that this article content has no conflict of interest.

\section{ACKNOWLEDGEMENT}

We thank Nasim for critical reading of the manuscript. This project was supported by General Logistics Department of Chinese People's Liberation Army (No. 20040207).

\section{REFERENCES}

[1] R. Avilés, J. Albizuri, A. Rodríguez, and L.N. López de Lacalle, "Influence of low-plasticity ball burnishing on the high-cycle fatigue strength of medium carbon AISI 1045 steel", Int. J. Fatigue, vol. 55, pp. 230-244, October 2013.

[2] Reference: Available from: http://www.ndt-ed.org/GeneralResourc es/MethodSummary/MethodSummary.htm

[3] S. Ghanei, M. Kashefi, and M. Mazinani, "Comparative study of eddy current and Barkhausen noise nondestructive testing methods in microstructural examination of ferrite-martensite dual-phase steel", J. Magn. Magn. Mater., vol. 356, pp. 103-110, January 2014 .

[4] A.A. Doubov, "A study of metal properties using the method of magnetic memory", Met. Sci. Heat Treat., vol. 39, pp. 695-701, September 1997.

[5] A.A. Doubov, "Diagnostics of metal items and equipment by means of metal magnetic memory", in Proc of CHSNDT 7th Conference on NDT and International Research Symposium, 1999, pp. 181-187.

[6] A.A. Doubov, "A technique for monitoring the bends of boiler and steam-line tubes using the magnetic memory of metal", Therm. Eng., vol. 48, pp. 289-295, August 2001.

[7] A.A. Doubov, "Diagnostics of equipment and constructions strength with usage of magnetic memory", Inspect. Diagnos., vol. 35, pp. 19-29, June 2001.

[8] L.H. Dong, B.S. Xu, S.Y. Dong, and Q.Z. Chen, "Metal magnetic memory testing for early damage assessment in ferromagnetic materials", J. Cent. South Univ. Technol., vol. 12, pp. 102-106, October 2005.

[9] Z.D. Wang, Y. Gu, and Y.S. Wang, "A review of three magnetic NDT technologies", J. Magn. Magn. Mater., vol. 32, pp. 382-388, February 2012.

[10] M. Roskosz, "Metal magnetic memory testing of welded joints of ferritic and austenitic steels", NDT E Int., vol. 44, pp. 305-310, May 2011.

[11] C.L. Shi, S.Y. Dong, B.S. Xu, and P. He, "Metal magnetic memory effect caused by static tension load in a case-hardened steel", $J$. Magn. Magn. Mater., vol. 322, pp. 413-416, February 2010.

[12] Z.D. Wang, K. Yao, B. Deng, and K.Q. Ding, "Theoretical studies of metal magnetic memory technique on magnetic flux leakage signals", NDT E Int., vol. 43, pp. 354-359, June 2010.

[13] X.L. Jian, X.C. Jian, and G.Y. Deng, "Experiment on relationship between the magnetic gradient of low-carbon steel and its stress", J. Magn. Magn. Mater., vol. 321, pp. 3600-3606, November 2009.

[14] L.J. Yang, B. Liu, L.J. Chen, and S.W. Gao, "The quantitative interpretation by measurement using the magnetic memory method (MMM)-based on density functional theory", NDT E Int., vol. 55, pp. 15-20, April 2013.

[15] M. Roskosz, and M. Bieniek, "Evaluation of residual stress in ferromagnetic steels based on residual magnetic field measurements", NDT E Int., vol. 45, pp. 55-62, January 2012. 
[16] K. Yao, B. Deng, and Z.D. Wang, "Numerical studies to signal characteristics with the metal magnetic memory-effect in plastically deformed samples", NDT E Int., vol. 47, pp. 7-17, April 2012.

[17] L.H. Dong, B.S. Xu, S.Y. Dong, Q.Z. Chen, and D. Wang, "Monitoring fatigue crack propagation of ferromagnetic materials with spontaneous abnormal magnetic signals", Int. J. Fatigue, vol. 30, pp. 1599-1605, September 2008.

[18] Z.D. Wang, K. Yao, B. Deng, and K.Q. Ding, "Quantitative study of metal magnetic memory signal versus local stress concentration", NDT E Int., vol. 43, pp. 513-518, September 2010.

(C) Gong et al.; Licensee Bentham Open.

This is an open access article licensed under the terms of the Creative Commons Attribution Non-Commercial License (http://creativecommons.org/licenses/ by-nc/3.0/) which permits unrestricted, non-commercial use, distribution and reproduction in any medium, provided the work is properly cited. 